\title{
In focus in $\mathrm{HCB}$
}

\author{
Douglas J. Taatjes $^{1}$ [ $~$ Jürgen Roth ${ }^{2}$
}

Accepted: 24 April 2018 / Published online: 4 May 2018

(c) Springer-Verlag GmbH Germany, part of Springer Nature 2018

\section{Blood-brain barrier computational pathology}

In the first of the review articles commemorating the 60th anniversary of Histochemistry and Cell Biology, Shityakov and Förster (2018) present a timely update on in silico modeling of the blood-brain barrier (BBB) in a variety of pathological circumstances. After initially describing some computational models employed to investigate BBB pathology in general, they then proceed to describe in individual sections specific computer models effective for the analysis of BBB issues, progressing from the molecular, to the cellular, tissue, and organ levels. Moreover, they provide a very informative table presenting a summary of the computational models currently in use at all levels of resolution relating to BBB pathology. The described models are based on mathematical principals and algorithms, in conjunction with multi-dimensional experimental data sets. Though much molecular information is currently available concerning proteins expressed at the $\mathrm{BBB}$, much remains to be learned concerning their alterations in pathological conditions, such as traumatic brain injury. The authors finish with a thoughtful synopsis on their perspectives for future areas and methods of investigation in this critical area of CNS pathology.

\section{Tryptase of mast cells}

The second review in this issue by Atiakshin et al. (2018) covers various aspects of the biogenesis and function of mast cell tryptase, the main component of their secretory

Douglas J. Taatjes

douglas.taatjes@uvm.edu

1 Department of Pathology and Laboratory Medicine, Larner College of Medicine, University of Vermont, Burlington, VT 05405, USA

2 University of Zurich, 8091 Zurich, Switzerland granules. Mast cells are migratory cells derived from the bone marrow and were discovered by Paul Ehrlich (reviewed by Buchwalow et al. 2015), which perform immunomodulatory tasks in health and disease. Following secretion, mast cell tryptase participates in the pathogenesis of a whole spectrum of diseased states including cardio-vascular, respiratory and digestive disorders, and in more general terms in the course of inflammatory processes where it may exert mainly pro-inflammatory and to a lesser extent anti-inflammatory effects. These aspects are discussed in great detail and provide an informative summary about the various roles secreted tryptase plays following activation of mast cells. The review is completed by aspects of the use of tryptase for diagnostic immunohistochemistry.

\section{Volume electron microscopy methods to study cardiomyocyte intercalated discs}

Three-dimensional imaging methods are required for investigating the complex morphological or ultrastructural aspects of biological structures. Volume-rendering imaging techniques such as confocal microscopy provide fine details of structures in multi-dimensions superior to those obtained with conventional widefield microscopy, yet still plagued by diffraction-limited resolution (Conchello and Lichtman 2006). Super-resolution microscopy techniques overcome the light microscopy diffraction limit, but still do not afford the superior resolution attained by electron microscopy (Klein et al. 2014). Thus, to perform a highresolution three-dimensional reconstruction of the intercalated disc in the myocardium, Vanslembrouck et al. (2018) turned to volume producing scanning electron microscopy (SEM) techniques. The intercalated disc is a specialized junctional complex connecting individual cardiomyocytes, and consisting of three specific membrane junctional complexes-desmosomes, adherens junctions, and gap junctions (Forbes and Sperelakis 1985), although the precise fine details of the disc structure are likely somewhat more 
complex. Using two different elegant high-resolution volume SEM techniques, serial block-face scanning electron microscopy (SBF-SEM) and focused ion beam SEM (FIB-SEM), together with an enhanced tissue staining and processing protocol, the authors visualized desmosomes and gap junctions in the mouse heart intercalated disc, and performed three-dimensional reconstructions (see cover figure) and morphometric measurements. Their results extend earlier EM observations by demonstrating (1) random spatial distribution of the junctions; (2) the complex folding pattern of the intercalated disc; (3) the close apposition of mitochondria to the discs; and (4) the presence of desmosomes and gap junctions in both plicae and interplicae subregions of the disc. The high-resolution and multi-dimensional imaging procedures described in this manuscript should prove as applicable for investigating potential alterations in intercalated disc ultrastructure occurring in cardiomyopathies.

\section{Interstitial cells of the urinary bladder: inter-species characteristics}

Interstitial cells (ICs) represent a substantial population of cells in the urinary bladder wall and have been proposed to fulfill various physiological roles including sensing the bladder filling state (Wiseman et al. 2003) or modulating afferent neuronal signals (Blyweert et al. 2004). Since animal models are commonly used to investigate urinary bladder physiology and pathophysiology, Steiner et al. (2018) have conducted a comparative immunohistochemical study of ICs in human, guinea pig and pig urinary bladder. They found that in all species $\geq 75 \%$ of ICs in the upper lamina propria (ULP) were positive for vimentin (VIM) and platelet-derived growth factor receptor alpha (PDGFR $\alpha$ ), and $\geq 90 \%$ positive for VIM and transient receptor potential cation channel A1 (TRPA1). In human and pig, $\geq 74 \%$ of ICS in the ULP were positive for VIM and alpha smooth muscle actin ( $\alpha \mathrm{SMA})$, while in guinea pig the percentage differed significantly with only $37 \%$ of ICs positive for VIM and $\alpha$ SMA. Additionally, over $90 \%$ of $\alpha$ SMA-positive ICs were also positive for TRPA1 and PDGFR $\alpha$ in human, guinea pig and pig. It is concluded that the pig bladder as compared to the guinea pig bladder provides a more suitable experimental model for studies on urinary bladder pathophysiology.

\section{References}

Atiakshin D, Buchwalow I, Samoilova V, Tiemann M (2018) Tryptase as a polyfunctional component of mast cells. Histochem Cell Biol. https://doi.org/10.1007/s00418-018-1659-8

Blyweert W, Aa F, Ost D, Stagnaro M, Ridder D (2004) Interstitial cells of the bladder: the missing link? BJOG 111(s1):57-60. https://doi. org/10.1111/j.1471-0528.2004.00469.x

Buchwalow I, Boecker W, Tiemann M (2015) The contribution of Paul Ehrlich to histochemistry: a tribute on the occasion of the centenary of his death. Virchows Arch 466:111-116

Conchello J-A, Lichtman JW (2006) Optical sectioning microscopy. Nat Methods 2:920-931

Forbes MS, Sperelakis N (1985) Intercalated discs of mammalian heart: a review of structure and function. Tissue Cell 14:25-37

Klein T, Proppert S, Sauer M (2014) Eight years of single-molecule localization microscopy. Histochem Cell Biol 141:561-575. https ://doi.org/10.1007/s00418-014-1184-3

Shityakov S, Förster C (2018) Computational simulation and modeling of the blood-brain barrier pathology. Histochem Cell Biol. https ://doi.org/10.1007/s00418-018-1665-x

Steiner C, Gevaert T, Ganzer R et al (2018) Comparative immunohistochemical characterization of interstitial cells in the urinary bladder of human, guinea pig and pig. Histochem Cell Biol. https ://doi.org/10.1007/s00418-018-1655-Z

Vanslembrouck B, Kremer A, Pavie B, van Roy F, Lippens S, van Hengel J (2018) Three-dimensional reconstruction of the intercalated disc including the intercellular junctions by applying volume scanning electron microscopy. Histochem Cell Biol. https://doi. org/10.1007/s00418-018-1657-x

Wiseman OJ, Fowler CJ, Landon DN (2003) The role of the human bladder lamina propria myofibroblast. BJU Int 91(1):89-93 